\title{
TRANSFER AND CHERN CLASSES FOR EXTRASPECIAL $p$-GROUPS
}

\author{
DAVID JOHN GREEN AND PHAM ANH MINH
}

\begin{abstract}
In the cohomology ring of an extraspecial $p$-group, the subring generated by Chern classes and transfers is studied. This subring is strictly larger than the Chern subring, but still not the whole cohomology ring, even modulo nilradical. A formula is obtained relating Chern classes to transfers.
\end{abstract}

\section{INTRODUCTION}

Methods to determine the cohomology ring of a finite group almost always presuppose that the cohomology of the Sylow $p$-subgroups is known. Calculating the cohomology ring of a $p$-group is however a delicate and difficult task. The extraspecial $p$-groups of exponent $p$ are in some sense the minimal difficult cases: minimal because their proper quotients are all elementary abelian, and their automorphism groups are very large. For this reason, many papers have been written, investigating their cohomology. Developments up till 1991 are surveyed in the paper [BC92]. In particular, M. Tezuka and N. Yagita obtained the prime ideal spectrum of the cohomology ring.

The usual method to calculate the cohomology of a $p$-group is to write the group as an extension, and solve the associated Lyndon-Hochschild-Serre spectral sequence. For the extraspecial $p$-groups however, such spectral sequences are intractable, and one is forced to look for other techniques. Now, standard constructions such as transfer (or corestriction) from subgroups and taking Chern classes of group representations provide us with a large number of cohomology classes. So many in fact, that for any $p$-group the classes provided by these two constructions generate a subring that has the same prime ideal spectrum as the cohomology ring (see GL96]). In this paper, we study this subring in the case of the extraspecial $p$-groups, and ask whether it is the whole cohomology ring.

Actually, these constructions yield very few odd-dimensional classes, and so it is rather more realistic to ask whether we obtain the whole cohomology ring modulo nilradical. At least for mod- $p$ cohomology, Proposition 9.4 answers this question too in the negative. For integral cohomology however, the problem remains open, and the significance of Corollary 8.2 is that this subring is the biggest studied to date in the cohomology ring of an extraspecial $p$-group.

This last result is proved using cohomology classes which we denote $\chi_{r, \phi}$. They are constructed in a manner foreshadowed in Min95. Take a product of Chern classes for the group of order $p^{2 n-1}$. By inflation and then corestriction, obtain a cohomology class $\chi_{r, \phi}$ for the group of order $p^{2 n+1}$. In Theorem 5.2, an elegant formula is obtained relating the Chern classes and the $\chi_{r, \phi}$, and in Theorem 7.2 we show that the $p^{\text {th }}$ power of any Chern class or any $\chi_{r, \phi}$ lies in the subring

Date: 3 March 1997.

1991 Mathematics Subject Classification. Primay 20J06; Secondary 20D15, 55R40.

The first author was supported by the Deutsche Forschungsgemeinschaft Schwerpunktprogramm "Algorithmische Zahlentheorie und Algebra".

The second author held a DAAD fellowship. 
generated by top Chern classes: this is the subring Tezuka and Yagita used to obtain the spectrum of the cohomology ring.

We are very grateful to Bruno Kahn for interesting discussions; and to Hélène Esnault and Eckhart Viehweg, who arranged for the second author to visit Essen.

\section{A Relation Between Dickson invariants}

We shall assume that the reader is familiar with the Dickson invariants: such familiarity may be acquired by consulting Benson's book Ben93, for example.

Let $\mathcal{V}$ be an $m$-dimension $\mathbb{F}_{p}$-vector space, and let $0 \leq r \leq m-1$. We shall write $D_{r}(\mathcal{V})$ for the Dickson invariant in degree $p^{m}-p^{r}$ in $S(\mathcal{V})$, or just $D_{m, r}$ if $\mathcal{V}$ is clear from the context. Recall that, for an indeterminate $X$, we have the equation

$$
V(\mathcal{V} ; X)=X^{p^{m}}+\sum_{r=0}^{m-1}(-1)^{m-r} D_{r}(\mathcal{V}) X^{p^{r}},
$$

where $V(\mathcal{V} ; X)$ in $S(\mathcal{V})[X]$ is defined by

$$
V(\mathcal{V} ; X)=\prod_{\phi \in \mathcal{V}}(X-\phi)
$$

The polynomial $V(\mathcal{V} ; X)$ is called the Mui invariant, as it is the most important of a family of invariants for subgroups of $G L_{m+1}$ studied by Mui Mui75. Note that $V(\mathcal{V} ; X)$ is $\mathbb{F}_{p}$-linear as a function of $X$. By convention, we define $D_{m, r}=0$ if $r<0$, and $D_{m, m}=1$.

Lemma 1.1. Let $\mathcal{V}$ be an $m$-dimensional $\mathbb{F}_{p}$-vector space, let $\mathcal{U}$ be a proper subspace of $\mathcal{V}$, and let $s$ be the codimension of $\mathcal{U}$ in $\mathcal{V}$. Let $\operatorname{Hyp}(\mathcal{V}, \mathcal{U})$ denote the set of all hyperplanes in $\mathcal{V}$ which contain $\mathcal{U}$ : this set is clearly nonempty. Then

$$
\sum_{\mathcal{W} \in \operatorname{Hyp}(\mathcal{V}, \mathcal{U})} V(\mathcal{W} ; X)=V(\mathcal{U} ; X)^{p^{s-1}}
$$

and it follows that

$$
\sum_{\mathcal{W} \in \operatorname{Hyp}(\mathcal{V}, \mathcal{U})} D_{r-1}(\mathcal{W})=D_{r-s}(\mathcal{U})^{p^{s-1}} \quad \text { for all } 1 \leq r \leq m .
$$

Proof. Let $\mathcal{T}$ be a complementary subspace of $\mathcal{U}$ in $\mathcal{V}$, so $\operatorname{dim}(\mathcal{T})=s$. The elements of $\operatorname{Hyp}(\mathcal{V}, \mathcal{U})$ are those subspaces $\mathcal{W}$ of $\mathcal{V}$ such that $\mathcal{W} \cap \mathcal{T}$ is a hyperplane in $\mathcal{T}$ and $\mathcal{W}=\mathcal{U} \oplus(\mathcal{W} \cap \mathcal{T})$. So choosing such a $\mathcal{T}$ induces a bijection between $\operatorname{Hyp}(\mathcal{V}, \mathcal{U})$ and the projective space $\mathbb{P} \mathcal{T}^{*}$ of lines in the dual space of $\mathcal{T}$.

Let $\tau_{1}, \ldots, \tau_{s}$ be a basis for $\mathcal{T}$. Observe that $V\left(\mathcal{U} ; \tau_{1}\right), \ldots, V\left(\mathcal{U} ; \tau_{s}\right)$, all homogeneous of degree $p^{n-s}$, are algebraically independent over $\mathbb{F}_{p}$ : for the image of $V\left(\mathcal{U} ; \tau_{i}\right)$ in $S(\mathcal{V} / \mathcal{U}) \cong S(\mathcal{T})$ is $\tau_{i}^{p^{n-s}}$.

Denote by $\underline{\lambda}$ an $s$-tuple $\left(\lambda_{1}, \ldots, \lambda_{s}\right)$ in $\mathbb{F}_{p}^{s}$. Given $\phi \in \mathbb{P} \mathcal{T}^{*}$, say that $\underline{\lambda}$ belongs to $\operatorname{ker}(\phi)$ if $\sum_{i=1}^{s} \lambda_{i} \tau_{i}$ does. Using Eqn. (1.2) and the linearity of $V(\mathcal{U} ;-)$, we obtain

$$
\begin{aligned}
\sum_{\mathcal{W} \in \operatorname{Hyp}(\mathcal{V}, \mathcal{U})} V(\mathcal{W} ; X) & =\sum_{\phi \in \mathbb{P}^{*}} \prod_{t \in \operatorname{ker}(\phi)} V(\mathcal{U} ; X-t) \\
& =\sum_{\phi} \prod_{\underline{\lambda} \in \operatorname{ker}(\phi)}\left(V(\mathcal{U} ; X)-\sum_{i=1}^{s} \lambda_{i} V\left(\mathcal{U} ; \tau_{i}\right)\right) .
\end{aligned}
$$

Consider the expression (1.6). It is a polynomial in $V(\mathcal{U} ; X), V\left(\mathcal{U} ; \tau_{1}\right), \ldots, V\left(\mathcal{U} ; \tau_{s}\right)$, and as such is homogeneous of degree $p^{s-1}$. Treat $V(\mathcal{U} ; X)$ as the main variable: then there is no constant term. Moreover, the coefficients are polynomials in $V\left(\mathcal{U} ; \tau_{1}\right), \ldots, V\left(\mathcal{U} ; \tau_{s}\right)$ which are invariant under the natural action of $G L_{s}\left(\mathbb{F}_{p}\right)$. 
So the coefficients must be polynomials in the Dickson invariants in these $s$ variables: but there are no such polynomials in positive degree less than $p^{s-1}$.

Therefore the coefficient in (1.6) of $V(\mathcal{U} ; X)^{j}$ is zero except when $j=p^{s-1}$. This coefficient is the size of $\mathbb{P}^{*}$, congruent to 1 modulo $p$.

\section{Chern Classes And extraspecial $p$-Groups}

The integral (group) cohomology of the unitary group $U(n)$ is a polynomial algebra with $n$ generators. A unitary representation of a finite group $G$ pulls these generators back to the integral cohomology of $G$; the images of the generators are the Chern classes of the representation. We refer the reader to Tho86] for more information about Chern classes and their properties.

Let $p$ be an odd prime. For $n \geq 1$, denote by $P=P_{n}$ the extraspecial $p$-group of order $p^{2 n+1}$ and exponent $p$. This fits into a central extension

$$
1 \rightarrow Z \rightarrow P_{n} \rightarrow E_{n} \rightarrow 1
$$

where $Z=Z(P)$ is cyclic of order $p$, and $E=E_{n}$ is elementary abelian of $p$ rank $2 n$. We may identify $Z$ with $\mathbb{F}_{p}$ and view $E$ as a $2 n$-dimensional $\mathbb{F}_{p}$-vector space: then the commutator map on $P$ induces a nondegenerate symplectic bilinear form $E \otimes_{\mathbb{F} p} E \rightarrow Z$.

Ignoring the $p^{2 n}$ linear characters, the remaining irreducible characters of $P$ all have degree $p^{n}$, and are distinguished by their restrictions to $Z$. Pick a nontrivial linear character $\gamma$ of $Z$, and define $\rho_{\gamma}$ to be the unique irreducible representation of $P$ whose restriction to $Z$ has character $p^{n} \gamma$. The other degree $p^{n}$ representations are then $\psi^{j}\left(\rho_{\gamma}\right)$ for $2 \leq j \leq p-1$.

Since $E$ is the abelianization of $P$, Chern classes of one-dimensional representations lie in the image of inflation from $E$ to $P$. The Chern classes of $\psi^{j}\left(\rho_{\gamma}\right)$ are scalar multiples of those of $\rho_{\gamma}$.

Pick an embedding of $\mathbb{F}_{p}$ in $\mathbb{C}^{\times}$: for each elementary abelian $p$-group $A$, this allows us to identify $\operatorname{Hom}\left(A, \mathbb{C}^{\times}\right)$with the dual space $A^{*}$.

The maximal elementary abelian subgroups $M$ of $P$ all have $p$-rank $n+1$, and are permuted transitively by the automorphism group of $P$. Each contains $Z$, and the map $M \mapsto I=M / Z$ induces a bijection between the $M$ and the maximal totally isotropic subspaces $I$ of $E$. Note that the dual $I^{*}$ is naturally a subspace of $M^{*}$.

View $\gamma$ as an element of $\mathrm{H}^{2}(Z, \mathbb{Z})$, since this isomorphic to $\operatorname{Hom}\left(Z, \mathbb{C}^{\times}\right)$. The restriction of $\rho_{\gamma}$ to $M$ decomposes as the direct sum of all representations whose restriction to $Z$ has character $\gamma$. The first Chern classes of these summands are all the classes in $\mathrm{H}^{2}(M, \mathbb{Z})$ whose restriction to $Z$ is $\gamma$; pick $\bar{\gamma}$ to be one of these first Chern classes, and observe that the kernel in $\mathrm{H}^{2}(M, \mathbb{Z}) \cong M^{*}$ of restriction to $Z$ is $I^{*}$. From the definition of $V$, the total Chern class of $\rho_{\gamma}$ therefore restricts to $M$ as $V\left(I^{*} ; 1+\bar{\gamma}\right)$, independent of the choice of $\bar{\gamma}$. Define $\zeta_{n} \in \mathrm{H}^{2 p^{n}}(P, \mathbb{Z})$ and, for $0 \leq r \leq n-1$, define $\kappa_{r}=\kappa_{n, r}$ in $\mathrm{H}^{2\left(p^{n}-p^{r}\right)}\left(P_{n}, \mathbb{Z}\right)$ by

$$
\zeta_{n}=c_{p^{n}}\left(\rho_{\gamma}\right) \quad \kappa_{n, r}=(-1)^{n-r} c_{p^{n}-p^{r}}\left(\rho_{\gamma}\right) .
$$

Then these are the only non-nilpotent Chern classes of $\rho_{\gamma}$, and

$$
\operatorname{Res}_{M}^{P} \zeta_{n}=V\left(I^{*} ; \bar{\gamma}\right) \quad \operatorname{Res}_{M}^{P}\left(\kappa_{r}\right)=D_{r}\left(I^{*}\right)
$$

It is useful, and sensible, to define $P_{0}$ to be $Z$ and $\zeta_{0}$ to be $\gamma$. Also to define $\kappa_{n, n}$ to be $1 \in \mathrm{H}^{0}(P, \mathbb{Z})$. 


\section{THE NEW CLASSES}

Since every group homomorphism $P \rightarrow \mathbb{F}_{p}$ factors through $E$, we may identify $E^{*}$ with the $\mathbb{F}_{p}$-vector space $\operatorname{Hom}\left(P, \mathbb{F}_{p}\right)$. Each maximal subgroup $H \leq P$ induces a one-dimensional subspace of $\operatorname{Hom}\left(P, \mathbb{F}_{p}\right)$, namely the subspace generated by any $\phi$ with $\operatorname{ker}(\phi)=H$. Consequently there is a natural bijection between the set of maximal subgroups of $P$ and the projective space $\mathbb{P} E^{*}$.

We are interested in the corestriction map from maximal subgroups to $P$. Let $\phi \in \mathbb{P} E^{*}$, and let $H=\operatorname{ker}(\phi)$ be the corresponding maximal subgroup of $P$. Then $H / Z$ is a $(2 n-1)$-dimensional $\mathbb{F}_{p}$-vector space, carrying a symplectic bilinear form with one-dimensional kernel. The centre $Z_{H}$ of $H$ is elementary abelian of $p$-rank 2 , and $Z_{H} / Z$ is the kernel of the form on $H / Z$. The maximal elementary abelian subgroups of $H$ all have $p$-rank $n+1$ and all contain $Z_{H}$. They are permuted transitively by the automorphism group of $H$.

Definition 3.1. For $n \geq 1$, for $0 \leq r \leq n-1$ and for $\phi \in \mathbb{P} E_{n}^{*}$, the class $\chi_{r, \phi}$ in $\mathrm{H}^{*}\left(P_{n}, \mathbb{Z}\right)$ with degree $2\left(p^{n}-p^{r}\right)$ is defined as follows. Set $H=\operatorname{ker}(\phi)$ and pick a rank one subgroup $A \neq Z$ of $Z_{H}$. Choosing such an $A$ induces a split epimorphism $H \rightarrow H / A \cong P_{n-1}$. Set

$$
\chi_{r, \phi}=\operatorname{Cor}_{H}^{P_{n}} \operatorname{Inf}_{P_{n-1}}^{H}\left(\kappa_{n-1, r} \zeta_{n-1}^{p-1}\right)
$$

Lemma 3.2. The class $\chi_{r, \phi}$ is well-defined. That is, it does not depend on the choice of $A \leq Z_{H}$.

Proof. The inner automorphisms of $P_{n}$ permute transitively all such subgroups $A \leq Z_{H}$, always fixing $Z$ pointwise and therefore sending the $\rho_{\gamma}$ for one $P_{n-1}$ to the $\rho_{\gamma}$ for the other. Since $H$ is normal in $P_{n}$ and corestriction commutes with conjugation, the result follows.

\section{Restriction AND THE NEW Classes}

In this section, we study restrictions of the classes $\chi_{r, \phi}$. We start however with a preparatory lemma.

Lemma 4.1. Let $\mathcal{V}$ be an $\mathbb{F}_{p}$-vector space, and let $\mathcal{U}$ be a hyperplane in $\mathcal{V}$. Pick $v$ in $\mathcal{V} \backslash \mathcal{U}$. Then the element $V(\mathcal{U} ; v)^{p-1}$ of $S(\mathcal{V})$ is invariant under all transformations of $\mathcal{V}$ which preserve $\mathcal{U}$. In particular, it is independent of the choice of $v$.

Proof. Any transformation which preserves $\mathcal{U}$ acts on the coset space $\mathcal{V} / \mathcal{U}$ as multiplication by some scalar in $\mathbb{F}_{p}^{\times}$. But, from its definition, $V(\mathcal{U} ; v)$ is invariant under all transformations which preserve $\mathcal{U}$ and the coset $v+\mathcal{U}$.

Definition 4.2. In the situation of Lemma 4.1, we denote by $V(\mathcal{U}, \mathcal{V})$ the invariant $V(\mathcal{U} ; v)^{p-1}$.

Lemma 4.3. Let $\mathcal{V}$ be an $\mathbb{F}_{p}$-vector space, and let $\mathcal{U}$ be a subspace with codimension two in $\mathcal{V}$. Then

$$
\sum_{\mathcal{W}} V(\mathcal{U}, \mathcal{W})=0
$$

where the sum is over all hyperplanes $\mathcal{W}$ in $\mathcal{V}$ which themselves contain $\mathcal{U}$ as a hyperplane.

Proof. Let $v, w$ be a basis for a subspace of $\mathcal{V}$ complementary to $\mathcal{U}$. Then

$$
\begin{aligned}
\sum_{\mathcal{W}} V(\mathcal{U}, \mathcal{W}) & =\sum_{[\lambda: \mu] \in \mathbb{F}_{p} \mathbb{P}} V(\mathcal{U} ; \lambda v+\mu w)^{p-1} \\
& =\sum_{[\lambda: \mu]}(\lambda V(\mathcal{U} ; v)+\mu V(\mathcal{U} ; w))^{p-1}
\end{aligned}
$$


This is an invariant of $G L_{2}\left(\mathbb{F}_{p}\right)$ acting on the rank 2 polynomial algebra generated by $V(\mathcal{U} ; v)$ and $V(\mathcal{U} ; w)$. Its degree in the generators is however $p-1$, and there are no invariants other than zero in this degree.

We now investigate the image of $\chi_{r, \phi}$ under restriction to each maximal elementary abelian $M \leq P$. If $M$ is contained in $H=\operatorname{ker}(\phi)$, then $Z_{H}$ is contained in $M$. Define $I_{\phi}$ to be the quotient $M / Z_{H}$. Then $I_{\phi}^{*}$ is a hyperplane in $I^{*}$, which is itself a hyperplane in $M^{*}$. Concretely, $I_{\phi}^{*}$ is the annihilator in $I^{*}$ of $Z_{H} / Z$ and also the annihilator in $M^{*}$ of $Z_{H}$.

Proposition 4.4. With the above notation, we have

$$
\operatorname{Res}_{M}\left(\chi_{r, \phi}\right)= \begin{cases}-D_{r}\left(I_{\phi}^{*}\right) V\left(I_{\phi}^{*}, I^{*}\right) & \text { if } M \leq \operatorname{ker}(\phi) \\ 0 & \text { otherwise. }\end{cases}
$$

Proof. Since $\chi_{r, \phi}$ was defined as a corestriction, we use the Mackey formula to determine its restriction to $M$. Both $M$ and $H$ are normal in $P$, and corestriction to $M$ from any proper subgroup is the zero map, at least in positive degree. This proves the result when $M$ is not contained in $H$. So we may now assume that $M \leq$ $H$.

As in the definition of $\chi_{r, \phi}$, choose a cyclic subgroup $A$ of $Z_{H}$ such that $Z_{H}=$ $A \times Z$. Then $M / A$ is a maximal elementary abelian subgroup of $H / A \cong P_{n-1}$. Note that $(M / A)^{*}$ is a hyperplane in $M^{*}$, and itself contains $I_{\phi}^{*}$ as a hyperplane: namely, the annihilator of $Z$. Then

$$
\begin{aligned}
\operatorname{Res}_{M}\left(\chi_{r, \phi}\right) & =\sum_{g \in P_{n} / H} g^{*} \operatorname{Res}_{M}^{H} \operatorname{Inf}_{P_{n-1}}^{H} \kappa_{n-1, r} \zeta_{n-1}^{p-1} \\
& =\sum_{g \in P_{n} / H} D_{r}\left(I_{\phi}^{*}\right) V\left(I_{\phi}^{*}, g^{*}(M / A)^{*}\right)
\end{aligned}
$$

Now, there were $p$ possible choices for $A$, each of which yields a different $(M / A)^{*}$. The possible $(M / A)^{*}$ are exactly those hyperplanes in $M^{*}$ which contain $I_{\phi}^{*}$ but are not equal to $I^{*}$. There are permuted faithfully and transitively by $P_{n} / H$. The result therefore follows from Eqn. (4.6) by Lemma 4.3.

\section{Describing Chern Classes in terms of COREstrictions}

The Chern classes $\kappa_{n, r}$ restrict to $Z$ as zero. A theorem of Carlson Eve91, $\S 10.2]$ says that any such class has some power which is a sum of corestrictions from proper subgroups. In this section we shall derive a formula for $\kappa_{n, r}$ in terms of the corestrictions $\chi_{r, \phi}$ and the image of inflation from $E$. First however, we recall a well-known fact about Dickson invariants.

Lemma 5.1. Let $\mathcal{V}$ be an $\mathbb{F}_{p}$-vector space, and $\mathcal{U}$ a hyperplane in $\mathcal{V}$. Then

$$
V(\mathcal{V} ; X)=V(\mathcal{U} ; X)^{p}-V(\mathcal{U} ; X) V(\mathcal{U}, \mathcal{V})
$$

and so, for $0 \leq r \leq n-1$,

$$
D_{r}(\mathcal{V})=D_{r-1}(\mathcal{U})^{p}+D_{r}(\mathcal{U}) V(\mathcal{U}, \mathcal{V})
$$

Here, recall that $D_{-1}(\mathcal{U})$ is zero.

Let $M_{0}$ be a maximal elementary abelian subgroup of $P$, and denote by $J_{0}$ the quotient $P / M_{0}$, itself elementary abelian of rank $n$. Then $J_{0}^{*}$ is the annihilator in $E^{*}$ of $M_{0}$. In addition, we may view $J_{0}^{*}$ as a subspace of $\mathrm{H}^{2}(E, \mathbb{Z})$. 
Theorem 5.2. For all $0 \leq r \leq n-1$, the degree $2\left(p^{n}-p^{r}\right)$ class $\nu_{r, M_{0}}$ in $\mathrm{H}^{*}(P, \mathbb{Z})$ defined by

$$
\nu_{r, M_{0}}=\kappa_{n, r}-\operatorname{Inf}_{E}^{P} D_{r}\left(J_{0}^{*}\right)+\sum_{\phi \in \mathbb{P} J_{0}^{*}} \chi_{r, \phi}
$$

is nilpotent.

Proof. By a theorem of Quillen [Eve91, Cor. 8.3.4], a class in the mod-p cohomology ring of a finite group is nilpotent if and only if the restriction to every elementary abelian $p$-subgroup is. Now, a class in integral cohomology reduces to zero in mod$p$ cohomology only if it is in the ideal generated by $p$. But in positive degree, all such classes are nilpotent. Hence it suffices to prove that the restriction to every elementary abelian subgroup is zero.

Let $M$ be a maximal elementary abelian subgroup of $P$. As before, define $I=$ $M / Z$, and $I_{\phi}=M /(Z(\operatorname{ker} \phi))$ for $\phi \in \mathbb{P} E^{*}$. Define $S$ to be the annihilator in $J_{0}^{*}$ of $M$, recalling that $J_{0}^{*}$ may be viewed as a space of homomorphisms $P \rightarrow \mathbb{F}_{p}$. Then $S=\left\{\phi \in E^{*} \mid M, M_{0} \in \operatorname{ker}(\phi)\right\}$.

If $S=\{0\}$, then $\operatorname{Res}_{M}\left(\chi_{r, \phi}\right)=0$ for every $\phi \in J_{0}^{*}$, and both $\kappa_{n, r}$ and $\operatorname{Inf}_{E}^{P} D_{r}\left(J_{0}^{*}\right)$ restrict to $M$ as $D_{r}\left(I^{*}\right)$. So $\operatorname{Res}_{M}\left(\nu_{r, M_{0}}\right)=0$ as required.

We may therefore assume that the $\mathbb{F}_{p}$-vector space $S$ has positive dimension $s$, which implies that the number of elements of the projective space $\mathbb{P} S$ is congruent to one modulo $p$. Denote by $R$ the subspace $\operatorname{Res}_{M} J_{0}^{*}$ of $I^{*}$, and observe that $S$ is the kernel of the quotient map $J_{0}^{*} \rightarrow R$. Now, $S$ contains by Proposition 4.4 the set of those $\phi \in J_{0}^{*}$ such that $\operatorname{Res}_{M}\left(\chi_{r, \phi}\right)$ is nonzero. Hence

$$
\begin{aligned}
\operatorname{Res}_{M}\left(\sum_{\phi \in \mathbb{P} J_{0}^{*}} \chi_{r, \phi}\right) & =-\sum_{\phi \in \mathbb{P} S} D_{r}\left(I_{\phi}^{*}\right) V\left(I_{\phi}^{*}, I^{*}\right) \quad \text { by Proposition } 4.4 \\
& =\sum_{\phi \in \mathbb{P} S}\left(D_{r-1}\left(I_{\phi}^{*}\right)^{p}-D_{r}\left(I^{*}\right)\right) \quad \text { by Lemma } 5.1 \\
& =D_{r-s}(R)^{p^{s}}-D_{r}\left(I^{*}\right) \quad \text { by Lemma } 1.1 \\
& =\operatorname{Res}_{M}\left(\operatorname{Inf}_{E}^{P} D_{r}\left(J_{0}^{*}\right)-\kappa_{n, r}\right) .
\end{aligned}
$$

Therefore $\operatorname{Res}_{M}\left(\nu_{r, M_{0}}\right)=0$, as desired.

\section{TAKING $p^{\mathrm{TH}}$ POWERS OF CHERn ClASSES}

In the next section we shall show that the $p^{\text {th }}$ powers of the new classes $\chi_{r, \phi}$ lie in the image of inflation from $E$, at least modulo nilradical. In preparation for this, we shall here prove the same result for the $p^{\text {th }}$ powers of the Chern classes $\kappa_{n, r}$.

Recall that $E$ carries a nondegenerate symplectic form, say $($,$) . Pick a symplectic$ basis $A_{1}, \ldots, A_{n}, B_{1}, \ldots, B_{n}$ for $E$ : so $A_{i} \perp A_{j}, B_{i} \perp B_{j}$ and $\left(A_{i}, B_{j}\right)=\delta_{i j}$. Let $\alpha_{1}, \ldots, \alpha_{n}, \beta_{1}, \ldots, \beta_{n}$ be the corresponding dual basis for $E^{*}$, which we recall may be identified with $\mathrm{H}^{2}(E, \mathbb{Z})$.

Definition 6.1. For $r \geq 1$, define $z_{n}^{(r)}$ in $S\left(E^{*}\right)$ by

$$
z_{n}^{(i)}=\sum_{i=1}^{n}\left(\alpha_{i} \beta_{i}^{p^{r}}-\alpha_{i}^{p^{r}} \beta_{i}\right) .
$$

By the work of Carlisle and Kropholler on symplectic invariants (see Ben93, $\S 8.3]$ ), the $z_{n}^{(i)}$ are invariant under symplectic transformations of $E$. In particular, this means that they do not depend upon the choice of symplectic basis. Tezuka and Yagita proved that, at least for $p$ odd, $z_{n}^{(1)}, \ldots, z_{n}^{(n)}$ is a regular sequence in $S\left(E^{*}\right)$; and that the ideal they generate contains every $z_{n}^{(i)}$ and is the intersection 
with $S\left(E^{*}\right)$ of the kernel of inflation from $\mathrm{H}^{*}(E, \mathbb{Z})$ to $\mathrm{H}^{*}(P, \mathbb{Z})$. (See BC92, Prop. 8.2 and $\S 10]$.)

Lemma 6.2. Let $x_{1}, \ldots, x_{m}$ be a regular sequence in a commutative ring $R$, and suppose that elements $a_{1}, \ldots, a_{m}$ of $R$ satisfy

$$
\sum_{i=1}^{m} a_{i} x_{i}=0
$$

Then each $a_{i}$ lies in the ideal generated by $x_{1}, \ldots, \hat{x}_{i}, \ldots, x_{m}$.

Proof. We note that the case $m=1$ is trivial, and proceed by induction on $m$. The product $a_{m} x_{m}$ lies in the ideal generated by $x_{1}, \ldots, x_{m-1}$, and therefore so does $a_{m}$ by regularity: say $a_{m}=\sum_{i=1}^{m-1} b_{i} x_{i}$. Defining $a_{i}^{\prime}=a_{i}+b_{i} x_{m}$ for $1 \leq i \leq m-1$, we have $\sum_{i=1}^{m-1} a_{i}^{\prime} x_{i}=0$. Thus we have reduced to the case of $m-1$, and the result follows by induction.

Corollary 6.3. Suppose that $f \in S\left(E^{*}\right)$ lies in the kernel of $\operatorname{Inf}_{E}^{P}$. Then there exist elements $f_{1}, \ldots, f_{n}$ of $S\left(E^{*}\right)$ such that $f=\sum_{i=1}^{n} f_{i} z_{n}^{(i)}$, and the images $\operatorname{Inf}_{E}^{P}\left(f_{i}\right)$ of the $f_{i}$ under inflation depend only on $f$, not on the choice of the $f_{i}$.

Proposition 6.4. There are unique classes $g_{0}, \ldots, g_{n-1}$ in $\mathrm{H}^{*}(P, \mathbb{Z})$ such that there exist $f_{0}, \ldots, f_{n-1}$ in $S\left(E^{*}\right)$ which satisfy both $g_{i}=\operatorname{Inf}_{E}^{P}\left(f_{i}\right)$ and

$$
z_{n}^{(n+1)}+\sum_{i=0}^{n-1}(-1)^{n-i} z_{n}^{(i+1)} f_{i}=0
$$

Each $g_{i}$ has degree $2\left(p^{n+1}-p^{i+1}\right)$, and $\kappa_{n, r}^{p}-g_{r}$ is nilpotent for all $0 \leq r \leq n-1$.

Proof. Existence and uniqueness of the $g_{i}$ follows from Corollary 6.3. We once again demonstrate nilpotence by proving that restriction to every maximal elementary abelian subgroup $M$ is zero.

Now, each $z_{n}^{(j)}$ is a symplectic invariant. Hence, for any symplectic transformation $\sigma$ of $E$, Corollary 6.3 says that Eqn. (6.3) still holds if each $f_{i}$ is replaced by $\sigma^{*}\left(f_{i}\right)$. Consequently, $\sigma^{*}$ fixes each $g_{i}$. As any linear transformation of a maximal totally isotropic subspace $I$ may be extended to a symplectic transformation of $E$, it follows that each $\operatorname{Res}_{M}\left(g_{i}\right)$ is a polynomial in the Dickson invariants for $I^{*}$. Since the symplectic transformations of $E$ permute the $I$ transitively, $\operatorname{Res}_{M}\left(g_{i}\right)$ is the same polynomial for each $M$. Finally, we see by comparing degrees that for each $i$ there is a scalar $\lambda_{i} \in \mathbb{F}_{p}$ such that $\operatorname{Res}_{M}\left(g_{i}\right)=\lambda_{i} D_{i}\left(I^{*}\right)^{p}$ for all $M$.

To establish the result from here, we have to prove that each $\lambda_{i}$ is 1 . First we shall prove that

$$
\phi^{p^{n+1}}+\sum_{i=0}^{n-1}(-1)^{n-i} \phi^{p^{i+1}} g_{i}=0 \quad \text { for all } \phi \in E^{*} \subseteq \mathrm{H}^{2}(P, \mathbb{Z}) .
$$

Since the $g_{i}$ are invariants for the symplectic group, it suffices to prove this for one nonzero $\phi$, say $\beta_{1}$. Recall that the inflation of $z_{n}^{(j)}$ is zero, and observe that differentiating $z_{n}^{(j)}$ with respect to $\alpha_{1}$ yields $\beta_{1}^{p^{j}}$. Consequently, differentiating both sides of Eqn. (6.3) with respect to $\alpha_{1}$ and then inflating yields Eqn. (6.4) with $\phi$ replaced by $\beta_{1}$. This establishes Eqn. (6.4).

Now restrict to any one maximal elementary abelian $M \leq P$ and take $p^{\text {th }}$ roots to obtain

$$
\phi^{p^{n}}+\sum_{i=0}^{n-1}(-1)^{n-i} \phi^{p^{i}} \lambda_{i} D_{i}\left(I^{*}\right)^{p}=0 \quad \text { for all } \phi \in I^{*} .
$$


This equation also holds for all $\phi \in I^{*}$ with each $\lambda_{i}$ replaced by 1 . Therefore each $\lambda_{i}$ must be 1 , or else taking the difference of the two left hand sides would yield a polynomial of degree less than $p^{n}$, with too many roots in an integral domain.

\section{TAKING $p^{\mathrm{TH}}$ POWERS OF THE NEW CLASSES}

There are $h_{1}, \ldots, h_{n-1}$ in $S\left(E_{n-1}^{*}\right)$ such that $z_{n-1}^{(n)}=\sum_{i=1}^{n-1} h_{i} z_{n-1}^{(i)}$, by Corollary 6.3. Now, $S\left(E_{n-1}^{*}\right)$ embeds in $S\left(E_{n}^{*}\right)$, and $z_{n}^{(i)}=z_{n-1}^{(i)}+z_{1}^{(i)}$. Here $z_{1}^{\prime(i)}$ signifies $z_{1}^{(i)}$ as a function of $\alpha_{n}, \beta_{n}$, that is $\alpha_{n} \beta_{n}^{p^{i}}-\alpha_{n}^{p^{i}} \beta_{n}$. So we have

$$
z_{n}^{(n)}=z_{1}^{\prime(n)}-\sum_{i=1}^{n-1} h_{i} z_{1}^{(i)}+\sum_{i=1}^{n-1} h_{i} z_{n}^{(i)}
$$

As $z_{1}^{\prime(i)}$ is divisible by $\beta_{n}$, there is a unique $\eta \in S\left(E^{*}\right)$ such that

$$
z_{n}^{(n)}=\beta_{n} \eta+\sum_{i=1}^{n-1} h_{i} z_{n}^{(i)}
$$

We may consider $\eta$ to be an element of $\mathrm{H}^{2 p^{n}}(E, \mathbb{Z})$.

Lemma 7.1. For every $\phi \in \mathbb{P} E^{*}$, the cohomology class $\chi_{n-1, \phi}^{p}$ lies in the image of $\operatorname{Inf}_{E}^{P}$ modulo nilpotent elements.

Proof. As the symplectic group permutes $\mathbb{P} E^{*}$ transitively, it is enough to prove the lemma for one $\phi$. We shall show that $\chi_{n-1, \beta_{n}}^{p}-\operatorname{Inf}_{E}^{P}(\eta)$ is nilpotent. Write $\chi$ for $\chi_{n-1, \beta_{n}}$.

Once more, we prove that the restriction of $\chi^{p}-\operatorname{Inf}_{E}^{P}(\eta)$ to every maximal elementary abelian $M \leq P$ is zero. The restriction of $\chi^{p}$ to each $M$ is known by Proposition 4.4. If the image of $\beta_{n}$ in $I^{*}$ is nonzero, then it follows from Eqn. (7.2) that $\operatorname{Res}_{M} \operatorname{Inf}^{P}(\eta)$ is zero. Henceforth we may assume that $M$ lies in the kernel of $\beta_{n}$, which is to say that $M$ contains $A_{n}$ and lies in $E_{n-1} \times\left\langle A_{n}\right\rangle$. Then $\operatorname{Res}_{M} \chi^{p}=$ $-V\left(I_{\beta_{n}}^{*} ; \alpha_{n}\right)^{p}$ by Proposition 4.4.

Differentiating Eqn. (7.2) with respect to $\beta_{n}$, then inflating to $P$ and finally restricting to $M$, we have

$$
-\alpha_{n}^{p^{n}}=\operatorname{Res}_{M} \operatorname{Inf}^{P}(\eta)-\sum_{i=1}^{n-1} \operatorname{Res}_{M} \operatorname{Inf}^{P}\left(h_{i}\right) \alpha_{n}^{p^{i}} .
$$

By Proposition 6.4 (for $P_{n-1}$ ) and Eqn. (1.1), the choice of the $h_{i}$ implies that

$$
\alpha_{n}^{p^{n}}-\sum_{i=1}^{n-1} \operatorname{Res}_{M} \operatorname{Inf}^{E}\left(h_{i}\right) \alpha_{n}^{p^{i}}=V\left(I_{\beta_{n}}^{*} ; \alpha_{n}\right)^{p},
$$

whence the result follows.

Theorem 7.2. For all $0 \leq r \leq n-1$ and all $\phi \in \mathbb{P} E^{*}$, the class $\chi_{r, \phi}^{p}$ lies in $\operatorname{Im}\left(\operatorname{Inf}_{E}^{P}\right)+\sqrt{0}$.

Proof. By a result of Evens [Eve68, Steenrod operations commute with corestriction. Therefore, at least in mod- $p$ cohomology, taking $p^{\text {th }}$ powers also commutes with corestriction. Hence Lemma 7.1 and Proposition 6.4 (applied to $P_{n-1}$ ) yield the result. 


\section{Linear indePENDENCE OF CORESTRICTIONS}

We have been looking at three subrings of the cohomology of the extraspecial $p$ groups. Firstly the subring generated by top Chern classes, then the Chern subring, and then the subring generated by top Chern classes and the corestrictions $\chi_{r, \phi}$. In Gre96, it is shown that top Chern classes generate a strictly smaller subring than the Chern subring, even modulo nilradical. Theorem 5.2 demonstrates that (at least modulo nilradical) the Chern subring is contained in the subring generated by top Chern classes and the $\chi_{r, \phi}$. This containment is now shown to be strict.

Proposition 8.1. Let $E^{\prime \prime}$ be a nondegenerate, codimension 2 subspace of E. Exactly $p+1$ elements $\phi \in \mathbb{P} E^{*}$ satisfy $E^{\prime \prime} \subseteq \operatorname{ker}(\phi)$. When $n=2$, no non-trivial $\mathbb{F}_{p}$-linear combination of these $\chi_{n-1, \phi}$ lies in $\operatorname{Im}\left(\operatorname{Inf}_{E}^{P}\right)+\sqrt{0}$.

Corollary 8.2. Assume $n=2$. Modulo nilradical, the Chern subring is strictly contained in the subring generated by top Chern classes and the $\chi_{r, \phi}$.

Proof of Corollary. Modulo nilradical, the degree $2\left(p^{2}-p\right)$ part of the Chern subring consists of the image of inflation together with one extra class, $\kappa_{1}$.

Let $E^{\prime}$ be the orthogonal complement of $E^{\prime \prime}$. Pick a symplectic basis for $E$ such that $E^{\prime}$ has basis $A_{1}, B_{1}$ and $E^{\prime \prime}$ has basis $A_{2}, B_{2}$. Note that each maximal isotropic subspace $I$ of $E$ satisfies either $I=\left(I \cap E^{\prime}\right) \oplus\left(I \cap E^{\prime \prime}\right)$ or $\left(I \cap E^{\prime}\right)=\left(I \cap E^{\prime \prime}\right)=0$.

Lemma 8.3. Consider the $x \in S\left(E^{*}\right)$ whose restriction to $S\left(I^{*}\right)$ is zero for every maximal totally isotropic $I \subseteq E$ satisfying $\left(I \cap E^{\prime}\right)=\left(I \cap E^{\prime \prime}\right)=0$. These $x$ form the ideal generated by $z_{2}^{(1)}$ and $\gamma_{2}=D_{1}\left(E^{\prime *}\right)-D_{1}\left(E^{\prime \prime *}\right)$.

Proof. Recall that $z_{2}^{(1)}, z_{2}^{(2)}$ form a regular sequence in $S\left(E^{*}\right)$, and generate the joint kernel of restriction to every $S\left(I^{*}\right)$. Observe that $\left(\alpha_{1} \beta_{1}^{p}-\alpha_{1}^{p} \beta_{1}\right) \gamma_{2} \in z_{2}^{(2)}+\left(z_{2}^{(1)}\right)$. Moreover, $\gamma_{2}$ has zero restriction to $S\left(I^{*}\right)$ if and only if $I$ satisfies $\left(I \cap E^{\prime}\right)=$ $\left(I \cap E^{\prime \prime}\right)=0$, whereas $\left(\alpha_{1} \beta_{1}^{p}-\alpha_{1}^{p} \beta_{1}\right)$ restricts to zero if and only if $I$ satisfies $I=\left(I \cap E^{\prime}\right) \oplus\left(I \cap E^{\prime \prime}\right)$. So the ideal in question is the ideal of $x$ such that $\left(\alpha_{1} \beta_{1}^{p}-\alpha_{1}^{p} \beta_{1}\right) x$ has zero restriction to every $S\left(I^{*}\right)$.

Proof of Proposition 8.1. Let $\phi \in \mathbb{P} E^{*}$ have $E^{\prime \prime} \subseteq \operatorname{ker}(\phi)$, and let $H$ be the corresponding maximal subgroup of $P$. For any maximal elementary abelian $M \leq H$, have that $I=M / Z$ satisfies $I=\left(I \cap E^{\prime}\right) \oplus\left(I \cap E^{\prime \prime}\right)$. By Proposition 4.4 it follows that $\operatorname{Res}_{M}\left(\chi_{1, \phi}\right)=0$ for all such $\phi$ and all $M$ satisfying $\left(I \cap E^{\prime}\right)=\left(I \cap E^{\prime \prime}\right)=0$. Invoking Lemma 8.3 and comparing degrees, the only element of $\operatorname{Im}\left(\operatorname{Inf}_{E}^{P}\right)$ that comes into consideration is $\gamma_{2}$ itself.

For no two of these $\phi$ do the corresponding maximal subgroups $H$ share a common maximal elementary abelian subgroup. Applying Proposition 4.4 again, these $\chi_{1, \phi}$ are therefore $\mathbb{F}_{p}$-linearly independent. Moreover, no nonzero restriction of a $\chi_{1, \phi}$ is a scalar multiple of the restriction of $\gamma_{2}$.

Remark 8.4. Proposition 8.1 and Corollary 8.2 can be proved in the same way for general $n \geq 2$, using the results of Gre96].

\section{Chern Classes AND TRANSFER NOT SUFFiCIENT}

Calculating cohomology rings of $p$-groups would be easier if there were a list of constructions, such as transfer and Chern classes, which together always yielded a set of generators for the cohomology ring. Being able to construct the cohomology ring modulo nilradical would be an important first step. This gave rise to the socalled $\overline{C h}$-conjecture, related to a construction of Moselle [Mos89]. We provide a counterexample to that conjecture. 
Definition 9.1. For a finite group $G$ and a prime $p$, the subring $\overline{C h}(G)$ of $\mathrm{H}^{*}\left(G, \mathbb{F}_{p}\right)$ is defined recursively as the subring generated by Chern classes for $G$, together with the images under corestriction of $\overline{C h}(H)$ for proper subgroups $H$ of $G$.

Remark 9.2. For $p=2$, one should use Stiefel-Whitney rather than Chern classes to get the largest possible subring.

Remark 9.3. Inclusion of $\overline{C h}(G)$ in $\mathrm{H}^{*}\left(G, \mathbb{F}_{p}\right)$ always induces an isomorphism of varieties: see GL96.

Proposition 9.4. Let $G$ be the extraspecial 3-group of order 27 and exponent 3 . Then $\overline{C h}(G) / \sqrt{0}$ is strictly contained in $\mathrm{H}^{*}\left(G, \mathbb{F}_{p}\right) / \sqrt{0}$.

Proof. Take a symplectic basis $A, B$ for $E=E_{1}$. We may view $A, B$ as elements of $P=P_{1}$. We shall consider restrictions to the four cyclic subgroups generated by $A, A B, A B^{2}, B$ respectively. For each of these four cyclic groups $K$, denote by $\xi$ the class in $\mathrm{H}^{1}\left(K, \mathbb{F}_{p}\right) \cong \operatorname{Hom}\left(K, \mathbb{F}_{p}\right)$ taking the generator to 1 . Let $x \in \mathrm{H}^{2}$ be the Bockstein of $\xi$.

For $p$-groups $H_{1}<H_{2}$, recall that $\operatorname{Cor}_{H_{1}}^{H_{2}} \operatorname{Res}_{H_{1}}^{H_{2}}$ is zero in mod- $p$ cohomology. In particular, $\mathrm{Cor}_{\mathrm{H}_{1}}^{\mathrm{H}_{2}}$ is zero whenever $\mathrm{H}_{2}$ is elementary abelian. Using the Mackey formula, it follows that $\operatorname{Res}_{K}^{P} \operatorname{Cor}_{H}^{P}$ is zero for any $H<P$ and for $K$ any of the four cyclic subgroups.

Let $\alpha, \beta$ be the dual basis for $E^{*}$. These may be viewed as elements of $\mathrm{H}^{1}\left(P, \mathbb{F}_{p}\right)$. Let $a, b \in \mathrm{H}^{2}$ be their Bocksteins. Then $a, b$ are also the first Chern classes of the corresponding representations, and all first Chern classes are in their span. Note that the restrictions of $a$ to the four cyclics are $x, x, x, 0$ respectively, whereas the restrictions of $b$ are $0, x,-x, x$ respectively.

We refer the reader to Lea92 for the fact that $\alpha \beta=0$ in $\mathrm{H}^{2}\left(P, \mathbb{F}_{p}\right)$, and for an account of Massey triple products in this context. Define $Y$ to be the Massey product $\langle\alpha, \alpha, \beta\rangle \in \mathrm{H}^{2}\left(P, \mathbb{F}_{p}\right)$. By naturality, this has zero restriction to the cyclics generated by $A, B$, whereas its restriction to the subgroup generated by $A B$ is $\langle\xi, \xi, \xi\rangle$. This is $-x$. Hence $Y$ lies outside $\overline{C h}(P)$, even modulo nilradical.

Remark 9.5. We conclude from the proof of Proposition 9.4 that any list of constructions that was always guaranteed to provide enough generators modulo nilradical for mod- $p$ cohomology would have to include Massey products.

Remark 9.6. Strictly, Proposition 9.4 is only a counterexample to the mod- $p$ version of the $\overline{C h}$-conjecture. One may however define in the same way a subring $\overline{C h}(G)$ of $\mathrm{H}^{*}(G, \mathbb{Z})$, and it is an open question whether containment modulo nilradical is strict in this case.

\section{REFERENCES}

[Ben93] D. J. Benson. Polynomial Invariants of Finite Groups. London Math. Soc. Lecture Note Ser. no. 190 (Cambridge Univ. Press, 1993).

[BC92] D. J. Benson and J. F. Carlson. The cohomology of extraspecial groups. Bull. London Math. Soc. 24 (1992), 209-235. Erratum: Bull. London Math. Soc. 25 (1993), 498.

[Eve68] L. Evens. Steenrod operations and transfer. Proc. Amer. Math. Soc. 19 (1968), 13871388 .

[Eve91] L. Evens. The Cohomology of Groups. (Oxford Univ. Press, 1991).

[Gre96] D. J. Green. "Chern classes and extraspecial groups of order $p^{5}$." Submitted for publication.

[GL96] D. J. Green and I. J. Leary. "The spectrum of the Chern subring." Submitted for publication.

[Lea92] I. J. Leary. The mod- $p$ cohomology rings of some p-groups. Math. Proc. Cambridge Philos. Soc. 112 (1992), 63-75.

[Mos89] B. Moselle. "Calculations in the cohomology of finite groups." Unpublished essay. Univ. of Cambridge, 1989. 
[Min95] P. A. Minh. Transfer map and Hochschild-Serre spectral sequences. J. Pure Appl. Algebra 104 (1995), 89-95.

[Mui75] H. Mui. Modular invariant theory and cohomology algebras of symmetric groups. J. Fac. Sci. Univ. Tokyo Sect. 1 A Math. 22 (1975), 316-369.

[Tho86] C. B. Thomas. Characteristic Classes and the Cohomology of Finite Groups. (Cambridge Univ. Press, 1986).

Inst. F. Exp. Math., Ellernstr. 29, D-45326 Essen, Germany

E-mail address: david@exp-math.uni-essen.de

Fachbereich 6 Mathematik, Universität Essen, D-45117 Essen, Germany

Current address: Department of Mathematics, University of Hue, Dai hoc Tong hop Hue, Hue, Vietnam 